\title{
CHARACTERIZATION OF HUMAN SKIN USING THz TIME DOMAIN SPECTROSCOPY FOR IN-BODY NANONETWORKS
}

\author{
Nishtha Chopra, Ke Yang, Mike \\ Philpott, Akram Alomainy \\ Queen Mary University of London, \\ Mile End Road, \\ London E1 4NS UK \\ n.chopra@qmul.ac.uk
}

\author{
Qammer H. Abbasi, Khalid \\ Qaraqe \\ Texas A\&M University at Qatar, \\ Engineering Building, Education City, \\ PO Box 23874, Doha, Qatar \\ \{qammer.abbasi; \\ khalid.qaraqe\}@qatar.tamu.edu
}

\begin{abstract}
In this paper, the intrinsic material properties of human skin via $\mathrm{THz}$ Time Domain Spectroscopy have been investigated in a working frequency range from 0.1-1.5 THz. These measured material parameters are utilized to characterize the channel for inbody nano-networks. The sample for spectroscopic study is the real human skin and repeated measurements have provided consistent refractive index and absorption coefficient results. The refractive index in the working frequency range is 1.5 , while abortion coefficient is $56 \mathrm{~cm}^{-1}$ at $1 \mathrm{THz}$.
\end{abstract}

\section{Keywords}

Nanonetworks, THz Time Domain Spectroscopy, Real Skin, Channel Capacity, Pathloss

\section{INTRODUCTION}

Nanocommunication at terahertz $(\mathrm{THz})$ frequency is proposed for a distributed network of nanomachines positioned inside the human body [1]. These devices are envisioned to communicate efficiently in the $\mathrm{THz}$ band of the spectrum thus making them as suitable candidates for healthcare applications. At the same time, the in/on/off-body radio communications have been extensively studied and characterized at microwave frequencies for a wide range of applications [2]. To date, the characterization of human skin is restricted to microwave and gigahertz frequency; a few samples have been measured in the THz range but provide sparse and inconsistent results $[3,4]$. To develop novel health monitoring systems, channel capacity has been calculated for lossy air media and presented that terahertz communications offer very high physical transmission rates, expected to be more than $1 \mathrm{~Tb} / \mathrm{s}$ and transmission distances on the order of a few tens of millimetres. Based on that study, pathloss for channel capacity was investigated and subdivided as absorption and spreading losses.
With the familiarity of the characteristics of the channel, a new physical layer aware medium access control (MAC) protocol, Time Spread On-Off Keying (TS-OOK), was proposed. To date, simulation results have been published for a free space medium emphasising that a communication link with acceptable signal to noise ratio can exist within the human tissue in the terahertz band [5]. To bridge this gap, the work presented here focuses on $\mathrm{THz}-$ Time Domain Spectroscopy (THz-TDS) of freshly excised real skin and a parameter extraction algorithm is applied to evaluate refractive index and absorption coefficient. With the growing interest in optical measurement and its technological advancements, new generation and detection schemes have paved the way for THz TDS. Many applications such as biological imaging, explosive detection and drug development have been reported [6]. The calculated parameters are then utilized for channel modelling of nanonetworks. The major part of any skin structure is the dermis and this is obtained from Blizard Institute, London UK and measured with the $\mathrm{THz}$ Time Domain Spectroscopy (THz-TDS) system at Queen Mary University of London.

\section{SUMMARY ON MEASUREMENT TECHNIQUE}

\subsection{THz TIME DOMAIN SPECTROSCOPY OF REAL SKIN}

Nonlinear optical phenomena such as optical rectification and the linear electro-optic effect have made broadband generation and detection of the $\mathrm{THz}$ radiation possible. With the handiness of $\mathrm{THz}$ Time Domain Spectroscopy (THz-TDS), we can obtain both phase and magnitude of a sample. The ultrafast laser pulses are focused via a set of off-axis parabolic mirrors on the real skin. The skin is mostly consisting of dermis, the second layer. Since the sample is freshly obtained and measured in two days of excision, the other layers such as fat and epidermis are rather challenging to preserve. The $\mathrm{THz}$ generation is possible via titanium-Sapphire laser which produces femtosecond pulses. These optical pulses are split with the aid of a beam splitter into two beams namely: pump and probe. Generation is made possible with the usability of photoconductive antenna illuminated by the pump beam resulting in a transient photocurrent. The pump beam travels through a time-delay stage resulting in a delay of $\Delta t$ relative to probe beam [2]. The samples are placed in a holder wedged between two TPX (Polymethylpentene) layers of 2.71 
mm thickness. The sample thickness is defined with the help of a spacer, which is fixed at $0.2 \mathrm{~mm}$ throughout the entire measurement. The fresh skin sample has high water content therefore after a few measurements on sample with various thicknesses from $0.2-1 \mathrm{~mm}$, a relatively low thickness value was an optimum choice. The pulsed laser - Ti: Sapphire has an adjustable wavelength range of 750-850 $\mathrm{nm}$; repetition rate of 80 $\mathrm{MHz}$ and peak power of about $1 \mathrm{~W}$.

\section{RESULTS \& DISCUSSION}

A simple transfer function based algorithm was used to extract the refractive index and absorption coefficient value. Further the pathloss equation for channel capacity [5] was used to illustrate the experimental verification. The measurements were performed in room temperature without nitrogen purging. The overall experimental setup is close to real-time situation, where a human body is surrounded by high absorbent atmosphere. TPX is an apt choice as a sample holder material as it is transparent to the incoming $\mathrm{THz}$ radiation.

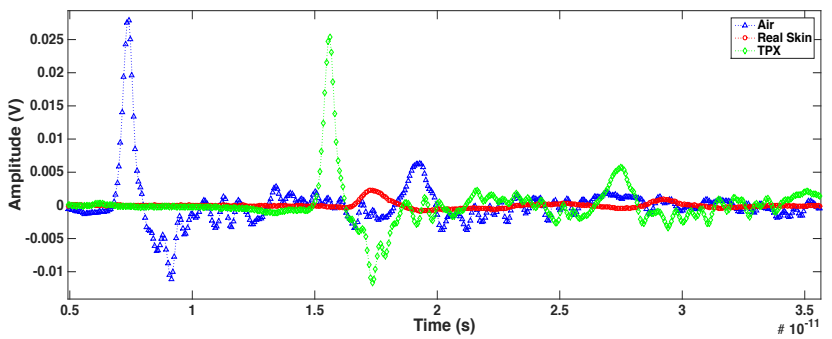

Fig. 1 The propagation of the THz wave through air, sample and TPX; the amplitude of the sample is attenuated due to increased absorption owing to high water content in the tissue.

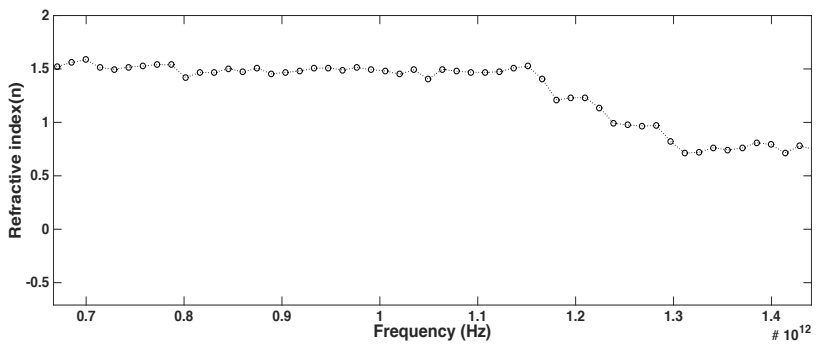

Fig. 2 The figure illustrates refractive index is 1.5 for a working frequency range of $0.7-1.2 \mathrm{THz}$ and thickness of the sample if fixed to $0.2 \mathrm{~mm}$.

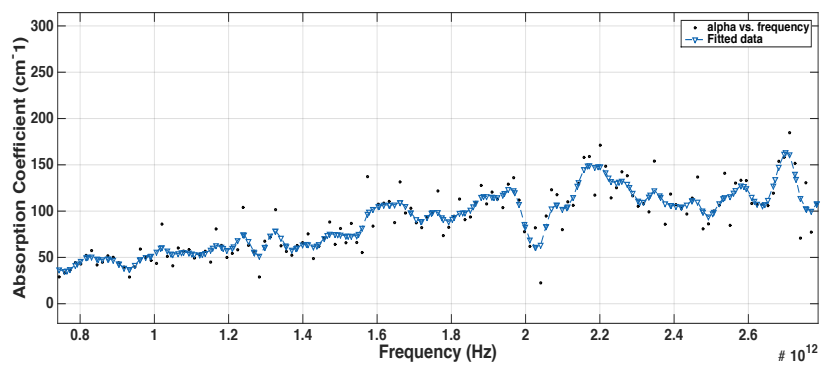

Fig. 3 Absorption coefficient of the sample around $1 \mathrm{THz} \&$ $1.2 \mathrm{THz}$ is $56 \mathrm{~cm}^{-1} \& 60 \mathrm{~cm}^{-1}$. The increase in value suggests that more incoming $\mathrm{THz}$ radiations gets absorbed at high frequencies.

\section{CONCLUSION}

The parameter extraction of real skin via $\mathrm{THz}$ Time Domain Spectroscopy provides a remarkable solution to fulfill the present sparse database in this frequency range with intricate information on skin composition and dielectric behavior. The paper has emphasized the need to generate such a database for various skin structures and orientations; this will be highlighted in the future work. The parameter values presented here are applied to calculate pathloss for channel capacity and further compared with a skin numerical model.

\section{ACKNOWLEDGMENTS}

Institute of Bioengineering PhD Scholarship, QMUL and Parts of this publication, specifically Section 3, were made possible by NPRP grant \# NPRP 7-125-2-061 from the Qatar National Research Fund (a member of Qatar Foundation).

\section{REFERENCES}

[1] I. F. Akyildiz, F. Brunetti, and C. Blázquez, "Nanonetworks: A new communication paradigm", Computer Networks, vol. 52, pp. 2260-2279, 2008.

[2] Chopra, N.; Yang, K.; Upton, J.; Alomainy, A.; Philpott, M.; Hao, Y., "Understanding and characterizing nanonetworks for healthcare monitoring applications," in RF and Wireless Technologies for Biomedical and Healthcare Applications (IMWS-Bio), 2014 IEEE MTT-S International Microwave Workshop Series on, vol., no., pp.1-3, 8-10 Dec. 2014

[3] S.Gabriel, R.W.Lau and C.Gabriel, "The dielectric properties of biological tissues: II. Measurements in the frequency range $10 \mathrm{~Hz}$ to $20 \mathrm{GHz}$ " Phys. Med. Biol. 41 (1996), 22512269.

[4] A. J. Fitzgerald and et al., "Catalogue of Human Tissue Optical Properties at Terahertz Frequencies," Journal of Biological Physics, vol. 9, pp. 123-128, 2003.

[5] Piro, G.; Ke Yang; Boggia, G.; Chopra, N.; Grieco, L.A.; Alomainy, A., "Terahertz Communications in Human Tissues at the Nanoscale for Healthcare Applications," in Nanotechnology, IEEE Transactions on, vol.14, no.3, pp.404-406, May 2015

[6] E. Berrya and et al., "Optical properties of tissue measured using terahertz pulsed imaging," in Proceedings of SPIE: Physics of Medical Imaging, 2003. 